\title{
Coronary sinus diameter by echocardiography to differentiate atrioventricular nodal reentrant tachycardia from atrioventricular reentrant tachycardia
}

\author{
Babu Ezhumalai, Santhosh Satheesh, Ajith Anantha, \\ Gobu Pakkirisamy, Jayaraman Balachander, Raja J. Selvaraj \\ Jawaharlal Institute of Postgraduate Medical Education and Research, Puducherry, India
}

\begin{abstract}
Background: Coronary sinus (CS) has been shown to be larger in patients with atrioventricular nodal reentrant tachycardia (AVNRT). We sought to determine if echocardiographically measured CS diameter can help identify the mechanism of tachycardia in patients with narrow complex tachycardia without preexcitation before the invasive electrophysiology study.

Methods: Forty four patients with documented narrow complex, short RP tachycardia who were scheduled for an electrophysiology study were included. Based on the electrophysiology stu$d y$, patients were divided into those with AVNRT and those with a concealed accessory pathway and atrioventricular reentrant tachycardia (AVRT). Proximal CS diameter (CSp) measured at the ostium and mid CS diameter (CSm) $1 \mathrm{~cm}$ distal to the ostium using transthoracic echocardiography.
\end{abstract}

Results: CSp was significantly larger in patients with AVNRT than AVRT (14.1 \pm 5 vs. $9.9 \pm 2 \mathrm{~mm}, p<0.0001)$. CSm diameter was not significantly different between the two groups. A cut-off of CSp $>11.2 \mathrm{~mm}$ identified AVNRT with a sensitivity of $92.6 \%$ and specificity of 76.9\%. CSp was a better discriminant (AUC 0.89, 95\% CI 0.75-0.97) compared to age (AUC 0.74, 95\% CI 0.58-0.87) or tachycardia rate (AUC 0.60, 95\% CI 0.44-0.76).

Conclusions: Echocardiographic measurement of the diameter of CS ostium can help in identifying the mechanism of the tachycardia before the invasive electrophysiology study. (Cardiol J 2014; 21, 3: 273-278)

Key words: coronary sinus, supraventricular tachycardia, echocardiography

\section{Introduction}

Narrow complex, short RP tachycardia without preexcitation in the sinus rhythm electrocardiogram (ECG) is a common clinical presentation. The primary differential diagnoses are atrioventricular nodal reentrant tachycardia (AVNRT) and atrioventricular reentrant tachycardia (AVRT) using a concealed accessory pathway. There are differences in the success and complication rates for ablative treatment of these arrhythmias making it desirable to identify the precise mechanism before the procedure.

Address for correspondence: Dr Raja Selvaraj, Assistant Professor, Department of Cardiology, JIPMER, Puducherry - 605006, India, tel: +91 9442242648, fax: +91 413 2272067, e-mail: rajajs@gmail.com

Received: 06.05.2013 Accepted: 15.05.2013 
Age of the patient, rate of tachycardia and presence of QRS alternans have some value in differentiating the mechanisms, but lack specificity and there is significant overlap between the two groups. History of neck pulsation, age at onset of symptoms and female sex have been shown to indicate the diagnosis of AVNRT [1-3]. Administration of adenosine or adenosine tri-phosphate has also been used to unmask accessory pathway conduction or dual atrioventricular nodal physiology [4-6]. Studies using venography have shown that the coronary sinus (CS) is larger and has a wind-sock shape in patients with AVNRT compared to controls $[7,8]$. Echocardiography is a simple and non-invasive investigation that is routinely performed in patients undergoing electrophysiology study. CS can be visualized and its diameter measured using echocardiography [9, 10]. Therefore, we investigated if echocardiographically measured CS diameter will differentiate patients with AVNRT from those with AVRT.

\section{Methods}

This is a prospective study carried out in a tertiary care hospital. Consecutive patients with documented narrow complex, short RP tachycardia who were scheduled for an electrophysiology study were included. Patients with preexcitation in the resting ECG were excluded. Informed consent was obtained from all participants. CS was visualized in the apical 4 chamber view [9] and the diameter was measured at 2 points by a trained cardiologist on the day before the electrophysiology study. Proximal CS diameter (CSp) was defined as the diameter at the ostium while the mid CS diameter (CSm) was defined as the diameter $1 \mathrm{~cm}$ distal to the ostium (Fig. 1). The morphological index was calculated as the ratio of the proximal to the mid CS diameters. A subgroup of measurements was repeated by a second observer and this was used to assess the inter-observer agreement of the measurements. During the subsequent electrophysiology study, diagnosis of the arrhythmia was made by an electrophysiologist based on standard criteria. Based on the diagnosis, the patients were divided into group I (final diagnosis of AVNRT) and group II (final diagnosis of AVRT).

\section{Statistical analysis}

Continuous variables were compared between the two groups using independent Student's $t$ test. Categorical variables were compared using the Fisher's exact test. Receiver operating charac-

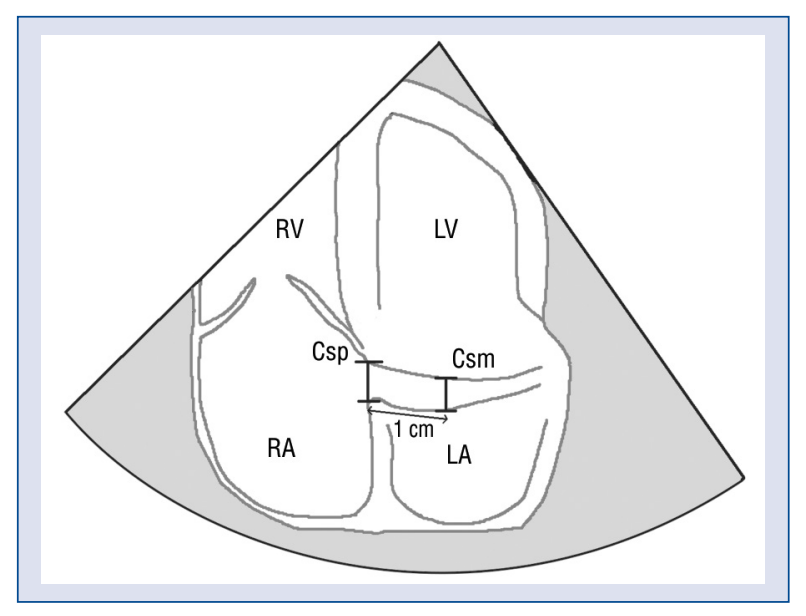

Figure 1. Echocardiographic measurement of coronary sinus (CS) diameter. Schematic diagram showing measurement of CS diameters by echocardiography. In the apical 4 chamber view with the probe tilted down, the CS can be made out and the diameter is measured at the ostium and $1 \mathrm{~cm}$ from the ostium; CSp - CS proximal diameter; CSm - CS mid diameter; LA — left atrium; LV — left ventricle; RA — right atrium; RV right ventricle.

teristic (ROC) curve was used to assess the sensitivity and specificity along with predictive values at different cut-off values of CS diameter in differentiating the two mechanisms. Area under the curve (AUC) was used as a measure of the diagnostic accuracy and $95 \%$ confidence intervals (CI) were used to quantify uncertainty. The interobserver agreement of echocardiographic measurement was assessed using intraclass correlation. A $\mathrm{p}<0.05$ was considered significant. Approval from the Institute Ethics Committee was obtained before commencing the study.

\section{Results}

\section{Demographics}

Forty four patients were included in the study. Of these, 30 patients had a final diagnosis of AVNRT (group I) and 14 patients had a final diagnosis of concealed accessory pathway with AVRT (group II). Patient characteristics in the two groups are listed in Table 1. Mean age was higher in group I. Tachycardia rate tended to be faster in group II, but this was not statistically significant.

\section{Echocardiography}

In 17 subjects, echocardiographic measurement of CSp and CSm was performed by 2 observers. These 34 measurements were used for asses- 
Table 1. Patient characteristics.

\begin{tabular}{lccc}
\hline & Group I (AVNRT, $\mathbf{n}=30)$ & Group II (AVRT, $\mathbf{n}=14)$ & P \\
\hline Age [years] & $48.7 \pm 11.6$ & $36.9 \pm 12.9$ & 0.004 \\
Males & $8(27 \%)$ & $6(43 \%)$ & 0.32 \\
Tachycardia rate $[\mathrm{bpm}]$ & $183 \pm 29$ & $198 \pm 23$ & 0.19 \\
\hline
\end{tabular}

AVNRT — atrioventricular nodal reentrant tachycardia; AVRT — atrioventricular reentrant tachycardia

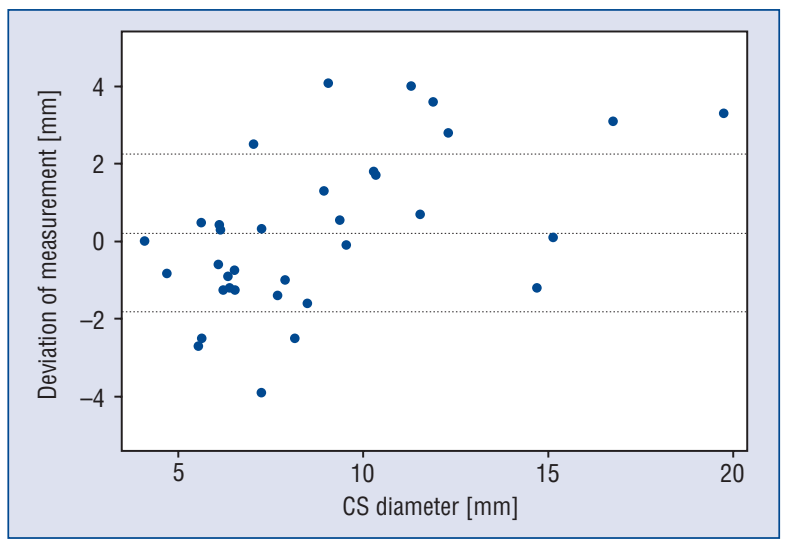

Figure 2. Coronary sinus (CS) diameter measurement — interobserver agreement; Bland Altman plot of agreement of CS measurements between 2 echocardiographers. There is a small bias, especially at larger diameters. Overall, the deviation of the measurements is small. Measurements and deviations are given in millimeters.

sing interobserver agreement. Mean measurement by operator 1 was $8.9 \pm 4.2 \mathrm{~mm}$ and by operator 2 was $8.7 \pm 3.2 \mathrm{~mm}$. Intra class correlation was 0.86 (95\% CI 0.73-0.93), suggesting good agreement of measurements (Fig. 2).

CS diameter could not be measured due to poor echocardiographic images in 4 of the $44(9 \%)$ patients, 3 in group I and 1 in group II. Representative echocardiographic images of the measurement from 2 patients in each group are shown in Figure 3. CSp was significantly larger in patients in group I (14.1 \pm 5 vs. $9.9 \pm 2$ mm, p < 0.001, Fig. 4). CSm was not significantly different $(7.2 \pm 2.5 \mathrm{~mm}$ for group I vs. $6.2 \pm 2.2 \mathrm{~mm}$ for group II, $\mathrm{p}=0.09$ ). The morphological index was also not significantly different between group I and group II $(2.1 \pm 0.8$ vs. $1.7 \pm 0.6, \mathrm{p}=0.09)$.

\section{Discrimination of tachycardia mechanisms}

Area under the ROC curve for diameter at CS ostium was 0.89 (95\% CI 0.75-0.97) as compared to the AUC for age $(0.74,95 \%$ CI $0.58-0.87)$ or tachycardia rate $(0.60,95 \%$ CI $0.44-0.76)$ (Fig. 5).
A cut-off of CSp $>11.2 \mathrm{~mm}$ identified AVNRT with a sensitivity of $92.6 \%$ and specificity of $76.9 \%$.

\section{Discussion}

We found that CS diameter at the ostium measured by echocardiography was significantly larger in patients with AVNRT compared to patients with AVRT. Diameter of the CS $1 \mathrm{~cm}$ from the ostium was not significantly different between the two groups. The ratio of the diameter at the ostium to the diameter at $1 \mathrm{~cm}$, the morphological index, which indicates the shape of the proximal CS, was not different between the two groups.

Doig et al. [8] measured the CS diameter in patients with AVNRT by venography and compared it to the diameter in controls. They found that the CS in patients with AVNRT was larger both at the ostium and at $5 \mathrm{~mm}$ and $10 \mathrm{~mm}$ from the ostium. The mean diameter at the ostium in the AVNRT group was $10.2 \mathrm{~mm}$, which is $2 \mathrm{~mm}$ less than that in our study and the diameter in controls was $8.1 \mathrm{~mm}$ which is $1.8 \mathrm{~mm}$ less than that in AVRT group in our study. This may represent a systematic difference of about $2 \mathrm{~mm}$ in CS measurement by echocardiography compared to venography, with the measurements in AVRT group being similar to controls. Doig et al. [8] also reported a windsock shape of the CSp, representing rapid tapering in a majority of patients with AVNRT while a tubular CS with minimal tapering was seen in most controls. We used the ratio of the CS ostium to the $\mathrm{CSm}$ as an index of the CS shape and did not find a significant difference between the two groups, although there was a trend towards a larger ratio in AVNRT. It is likely that the tapering of the CS occurs beyond $1 \mathrm{~cm}$ as mentioned by Doig et al. [8] and therefore, the ratio measured at $1 \mathrm{~cm}$ did not represent this shape.

We did not find measurement of CS diameter by transthoracic echocardiography technically challenging. Adequate measurements could be obtained in $90 \%$ of the patients. There was a good interobserver agreement of measurements between the 2 different cardiologists. 


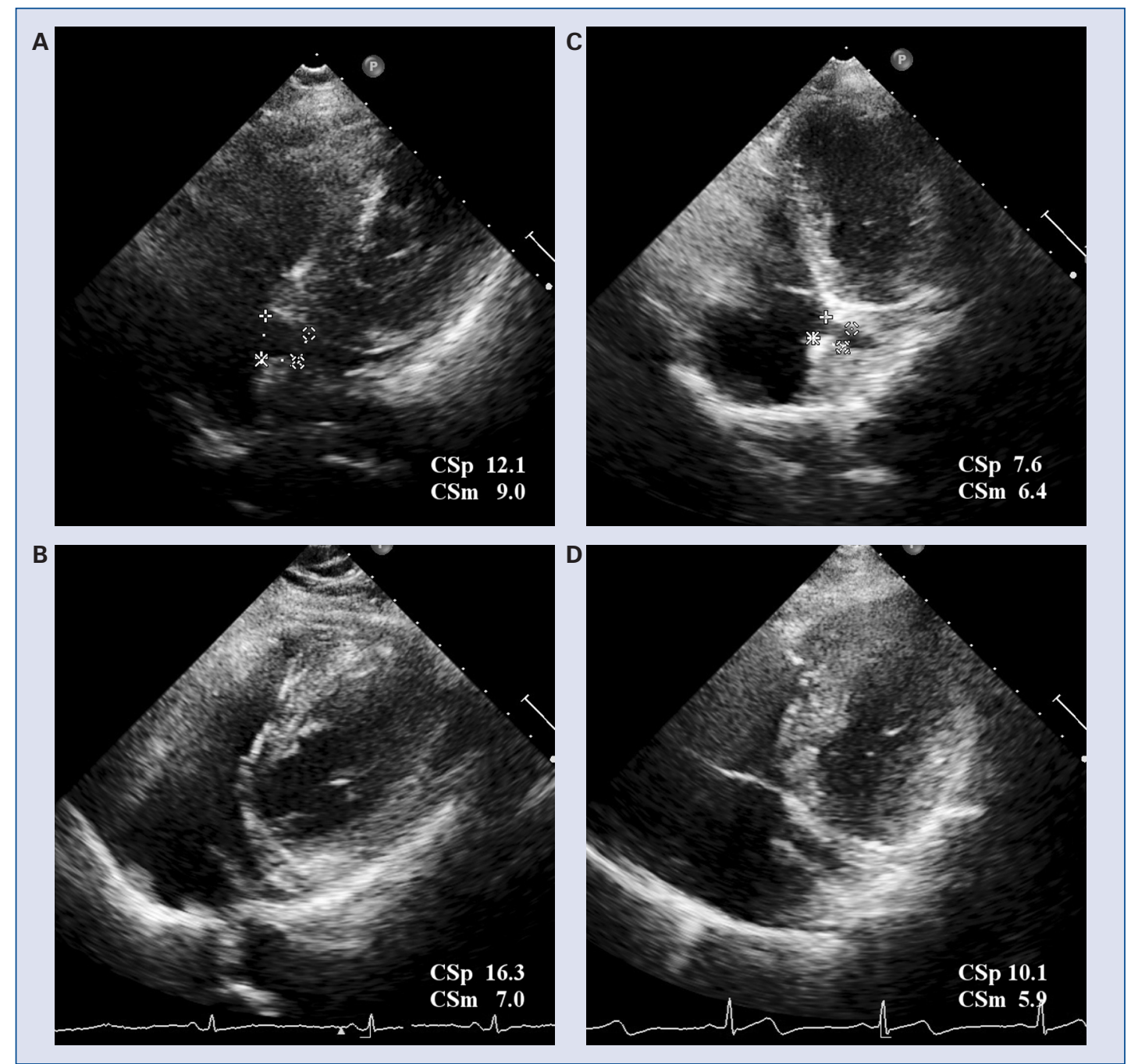

Figure 3. Illustrative echocardiographic coronary sinus (CS) images. Each panel shows the coronary sinus as visualized in a modified apical 4 chamber view in a different patient. Panels $\mathbf{A}$ and $\mathbf{B}$ are from patients in group I (AVNRT) while panels $\mathbf{C}$ and $\mathbf{D}$ are from patients in group II (AVRT). Measurements at the proximal coronary sinus (CSp) and the mid coronary sinus (CSm) given in millimetres are shown in each panel.

Various studies have attempted to analyze variables differentiating the mechanism in patients with narrow complex tachycardia. It is recognized that patients with AVNRT are generally older [11, $12]$, although there is a significant overlap between the groups making this fact less clinically useful. History of neck pulsations has been proposed as a strong indicator of AVNRT [1], but in our experience this is highly subjective and unreliable. ECG recorded during tachycardia can give various clues, including presence of a pseudo r' wave in $\mathrm{V}_{1}$ and pseudo $\mathrm{s}$ wave in inferior leads, ST depression [13-17], ST elevation in AVR [15, 18] or QRS alternans $[15,16,19,20]$. However, these findings have a low sensitivity, are sometimes subjective and, most importantly, require ECG documentation during tachycardia, which may not be available.
Adenosine administration can unmask pathway conduction in patients with latent preexcitation and demonstrate dual AV nodal conduction in some patients with AVNRT [4] with a sensitivity of $74 \%$, but is cumbersome to perform.

Our findings are consistent with previous reports of an enlarged CS ostium in patients with AVNRT. The exact mechanism underlying the association of an enlarged CS ostium and AVNRT is unknown. Jackman et al. [21] have proposed a circuit for typical slow-fast AVNRT in which retrograde conduction reaches the left atrium by the fast pathway and then proceeds sequentially along the CS roof and floor to reach the slow pathway region. Thus, CS myocardium forms part of the AVNRT circuit [21]. Stretching can alter myocardial conduction properties $[8,22,23]$ and it is possible 


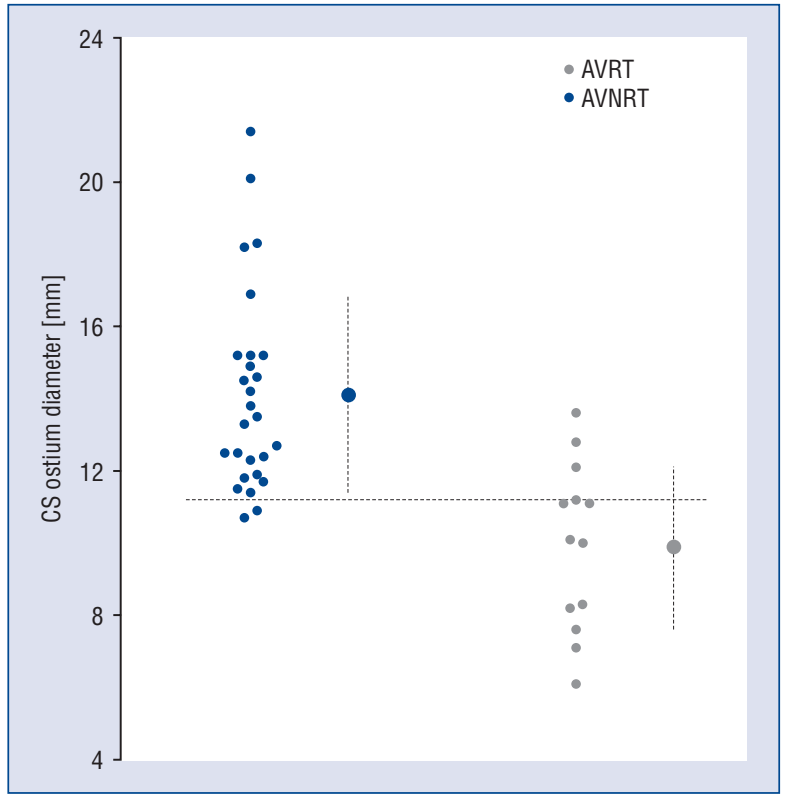

Figure 4. Coronary sinus (CS) diameter at ostium in the two groups. Diameter at the CS ostium measured by echocardiography in patients with atrioventricular nodal reentrant tachycardia (AVNRT; blue circles) and with atrioventricular reentrant tachycardia (AVRT; gray circles). Mean and one standard deviation on either side of the mean are also shown. The broken line represents the optimal cut off of $11.2 \mathrm{~mm}$.

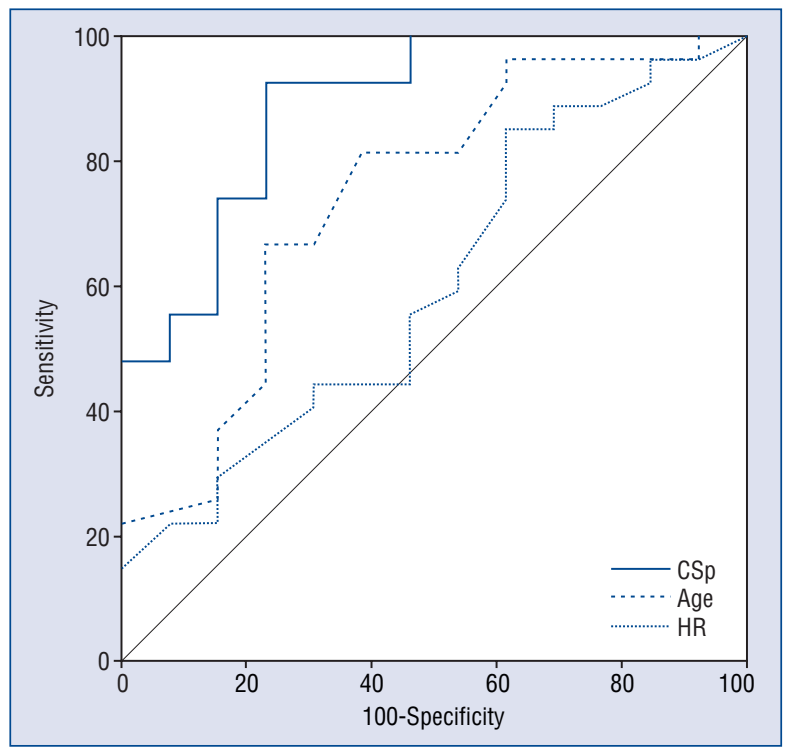

Figure 5. Receiver operator characteristics (ROC) curves for discrimination of tachycardia mechanism; ROC curves are shown for age, tachycardia rate (HR) and coronary sinus diameter at ostium (CSp) for differentiating patients with atrioventricular nodal reentrant tachycardia (AVNRT) from those with atrioventricular reentrant tachycardia (AVRT). that stretching the slow pathway can make a dual AV nodal physiology manifest and result in AVNRT in these patients.

\section{Limitations of the study}

We did not attempt to study other variables like history of neck pulsations and findings in the ECG. However, history of neck pulsations tends to be unreliable and subjective, while ECGs are not available in all patients. A systematic analysis of presence of dual AV nodal physiology in the patients with AVRT was not performed. This could help understand the mechanism and support the stretch hypothesis if patients with dual AV nodal physiology had a larger CS ostium. Finally, the number of patients included in the study is small, although the results clearly support the validity of CS diameter as a discriminant of mechanisms.

\section{Conclusions}

Echocardiographic measurement of CS ostium can help differentiate patients with AVNRT from those with AVRT among those presenting with narrow complex tachycardia. Patients with AVNRT have a larger diameter of the CS ostium compared to patients with AVRT. This can serve as a simple, non-invasive method to identify the tachycardia mechanism before the electrophysiology procedure.

Conflict of interest: none declared

\section{References}

1. Gonzalez-Torrecilla E, Almendral J, Arenal A et al. Combined evaluation of bedside clinical variables and the electrocardiogram for the differential diagnosis of paroxysmal atrioventricular reciprocating tachycardias in patients without pre-excitation. J Am Coll Cardiol, 2009; 53: 2353-2358.

2. Rodriguez LM, de Chillou C, Schlapfer J et al. Age at onset and gender of patients with different types of supraventricular tachycardias. Am J Cardiol, 1992; 70: 1213-1215.

3. Gursoy S, Steurer G, Brugada J, Andries E, Brugada P. The hemodynamic mechanism of pounding in the neck in atrioventricular nodal re-entrant tachycardia. N Engl J Med, 1992; 327: 772- 774.

4. Toal SC, Vajifdar BU, Gupta AK, Vora AM, Lokhandwala YY. Adenosine induced PR jump on surface ECG to differentiate atrioventricular nodal re-entrant tachycardia from concealed accessory pathway mediated tachycardia: A bedside test. Heart, 2002; 87: 37-40.

5. Camm AJ, Garratt CJ. Adenosine and supraventricular tachycardia. N Engl J Med, 1991; 325: 1621-1629.

6. Belhassen B, Fish R, Glikson M et al. Non-invasive diagnosis of dual AV node physiology in patients with AV nodal reentrant tachycardia by administration of adenosine 5 triphophaste during sinus rhythm. Circulation, 1998; 98: 47-53. 
7. Weiss C, Cappato R, Willems S, Meinertz T, Kuck KH. Prospective evaluation of the coronary sinus anatomy in patients undergoing electrophysiologic study. Clin Cardiol, 1999; 22: 537-543.

8. Doig JC, Saito J, Harris L, Downar E. Coronary sinus morphology in patients with atrioventricular junctional reentry tachycardia and other supraventricular tachyarrhythmias. Circulation, 1995; 92: 436-441.

9. Kronzon I, Tunick PA, Jortner R et al. Echocardiographic evaluation of the coronary Sinus. J Am Soc Echocardiog, 1995; 8: 518-526.

10. Gunes Y, Guntekin U, Tuncer M, Kaya Y, Akyol A. Association of coronary sinus diameter with pulmonary hypertension. Echocardiography, 2008; 25: 935-940.

11. Denes P, Wu D, Dhingra R, Amat-y-Leon F, Wyndham C, Rosen KM. Dual atrioventricular nodal pathways: A common electrophysiological response. Br Heart J, 1975; 37: 1069-1076.

12. Clague JR, Dagres N, Kottkamp H, Breithardt G, Borggrefe M. Targeting the slow pathway for atrioventricular nodal reentrant tachycardia: Initial results and long-term follow-up in 379 consecutive patients. Eur Heart J, 2001; 22: 82-88.

13. Arya A, Kottkamp H, Piorkowski C et al. Differentiating atrioventricular nodal reentrant tachycardia from tachycardia via concealed accessory pathway. Am J Cardiol, 2005; 95: 875-878.

14. Riva SI, Della Bella P, Fassini G, Carbucicchio C, Tondo C. Value of analysis of ST segment changes during tachycardia in determining type of narrow QRS complex tachycardia. J Am Coll Cardiol, 1996; 27: 1480-1485.

15. Letsas KP, Weber R, Siklody $\mathrm{CH}$ et al. Electrocardiographic differentiation of common type atrioventricular nodal reentrant tachycardia from atrioventricular reciprocating tachycardia via a concealed accessory pathway. Acta Cardiol, 2010; 65: 171-176.

16. Erdinler I, Okmen E, Oguz E, Akyol A, Gurkan K, Ulufer T. Differentiation of narrow QRS complex tachycardia types using the 12-lead electrocardiogram. Ann Noninvasive Electrocardiol, 2002; 7: 120-126.

17. Fassini G, Riva S, Della Bella P, Carbucicchio C, Tondo C. Analysis of changes in repolarization for the differential diagnosis of narrow QRS supraventricular tachycardia and the site of the accessory pathway. Cardiologia, 1996; 41: 869-875.

18. Zhong YM, Guo JH, Hou AJ, Chen SJ, Wang Y, Zhang HC. A modified electrocardiographic algorithm for differentiating typical atrioventricular node re-entrant tachycardia from atrioventricular reciprocating tachycardia mediated by concealed accessory pathway. Int J Clin Pract, 2006; 60: 1371-1377.

19. Green M, Heddle B, Dassen W et al. Value of QRS alternation in determining the site of origin of narrow QRS supraventricular tachycardia. Circulation, 1983; 68: 368-373.

20. Morady F, DiCarlo LA Jr, Baerman JM, de Buitleir M, Kou WH. Determinants of QRS alternans during narrow QRS tachycardia. J Am Coll Cardiol, 1987; 9: 489-499.

21. Jackman WM, Lockwood D, Nakagawa H et al. Chapter 9: Catheter ablation of AV nodal reentrant tachycardia. In: Wilber DJ, Packer DL, Stevenson WG eds. Catheter ablation of cardiac arrhythmias: Concepts and clinical applications. $3^{\text {rd }}$ Ed. Blackwell/ /Futura Publishing, Oxford, UK, 2007.

22. Reiter MJ, Synhorst DP, Mann DE. Electrophysiological effects of acute ventricular dilatation in the isolated rabbit heart. Circ Res, 1988; 62: 554-562.

23. Lab MJ. Contraction-excitation feedback in myocardium: Physiological basis and clinical relevance. Circ Res, 1982; 50: 757-766. 